$\begin{array}{cl}\begin{array}{cl}\text { Revue } \\ \text { de Ihistoire }\end{array} & \text { Revue de l'histoire des religions } \\ \text { des religions } & 4 \mid 2010 \\ & \text { Qu'est-ce qu'un « paysage religieux » }\end{array}$

\title{
Lavinium, Alba Longa, Roma : à quoi sert un paysage religieux?
}

Lavinium, Alba Longa, Roma: why a religious landscape?

\section{Alexandre Grandazzi}

\section{(2) OpenEdition}

\section{Journals}

Édition électronique

URL : http://journals.openedition.org/rhr/7673

DOI : $10.4000 /$ rhr.7673

ISSN : 2105-2573

Éditeur

Armand Colin

Édition imprimée

Date de publication : 1 décembre 2010

Pagination : $573-590$

ISBN : 978-2200-92658-8

ISSN : 0035-1423

Référence électronique

Alexandre Grandazzi, "Lavinium, Alba Longa, Roma : à quoi sert un paysage religieux ? », Revue de

I'histoire des religions [En ligne], 4 | 2010, mis en ligne le 17 février 2011, consulté le 19 avril 2019. URL: http://journals.openedition.org/rhr/7673; DOI : 10.4000/rhr.7673 


\section{ALEXANDRE GRANDAZZI}

Université Paris IV-Sorbonne

Équipe d'accueil Édition et commentaire des textes grecs et latins (E.A.1491)

\section{Lavinium, Alba Longa, Roma : à quoi sert un paysage religieux?}

Est analysé le parcours cérémoniel effectué par les consuls romains à leur entrée en charge : Capitole, mont Albain, Lavinium. Il s'agit d'un système spatio-rituel, constitutif en tant que tel d'un paysage religieux: on peut y déchiffrer, sous la République et l'Empire, le lien établi par le pouvoir romain entre un discours sur les origines de l'Urbs et une définition ouverte de la citoyenneté. Différents indices permettent de dater la mise au point de ce système, où mythe et rite, temps et espace se répondent exactement. Le paysage religieux du suburbium a été ainsi construit comme une métaphore de l'identité romaine.

\section{Lavinium, Alba Longa, Roma : why a religious landscape?}

The ceremonial procedure is analysed by the Roman Consuls as they take office: Capitol, Alban Mount, Lavinium. It is a system determined according to a ritual of time and place, such as a religious ritual : Under the Republic and the Empire, one can decipher the link established by the powers of Rome between a speech on the origins of the Urbs and an open definition of citizenship. Various indications allow us to date the adjustment of this system, where myth and ritual, time and space, are matched. The religious landscape of suburbium was therefore constructed as a metaphor of Roman identity. 


\section{UN PARCOURS CÉRÉMONIEL}

À Rome, sous la République et sous l'Empire, la coutume et la religion - dont la conjonction équivaut un peu à ce qu'est pour nous le droit constitutionnel - voulaient qu'un consul nouvellement élu eût à accomplir, hors de la Ville, toute une pérégrination religieuse. Préalablement, il avait d'abord dû monter sur la plus escarpée des collines romaines, le Capitole, pour y accomplir un sacrifice, prononcer une prière publique et réunir une première fois le Sénat afin que cette assemblée fixât notamment le jour où serait célébrée une autre cérémonie : les Fêtes Latines, qui se tiendraient sur le mont Albain, à une trentaine de kilomètres au sud-est de l'Urbs. Une dizaine de jours après ces festivités, il lui faudrait encore partir pour Lavinium, où il sacrifierait à Vesta et aux Dieux Pénates du peuple romain ${ }^{1}$. Capitole, mont Albain, Lavinium : ces trois lieux dessinent ainsi, puisque sur chacun d'eux des rites spécifiques doivent être célébrés, un paysage religieux, qu'il convient de regarder et de déchiffrer comme tel, dans toutes ses significations.

Pour ce faire, on dressera d'abord un état des lieux - religieux, institutionnel et topographique -, avant de passer à une double analyse : en synchronie, sera étudié le fonctionnement du système ainsi mis en lumière pour une époque correspondant in globo à la fin de la République et au principat julio-claudien; l'enquête diachronique, elle, fera apparaître les causes et les objectifs auxquels a pu répondre la constitution de ce que j'appellerai dès maintenant un système spatio-rituel.

Les premières caractéristiques d'un tel système sont la répétition et l'obligation : en effet, ce parcours cérémoniel est à recommencer chaque année par tout nouveau consul. La preuve par défaut en est fournie par l'anecdote de Flaminius, partant, sitôt élu, pour la guerre

1. Les différents textes qui attestent l'existence de chacune des étapes ici résumées sont analysés par Theodor Mommsen, Droit Public Romain, trad. Paul Frédéric Girard, t. II, Paris [1892] 1984, p. 287-290 : pour celle du Capitole, il s'agit de la uotorum nuncupatio et du s.c. de feriis Latinis (Cicéron, ad fam., VIII, 6, 3). C'est ce que Mommsen appelle « les formalités de l'entrée en fonctions », « [die] Formalien des Amtsantritts »: Römisches Staatsrecht, Leipzig, $1876^{2}$, t. I, p. 597. 
contre Hannibal, sans passer par le Capitole ni par le mont Albain². Entre ce manquement rituel du consul trop pressé et le désastre de Trasimène qui s'ensuivit, avec la mort dudit Flaminius, l'annalistique établit clairement un rapport de cause à effet ${ }^{3}$, à tel point qu'on a bien l'impression d'avoir affaire à l'étiologie, historisante comme toujours à Rome, de cette obligation religieuse. La preuve par excès est apportée par les onze jours que César, pourtant très pressé d'aller à la poursuite de Pompée, passe à Rome, en 49 avant J.-C., afin de célébrer les Fêtes Latines et d'accomplir le sacrifice à Lavinium ${ }^{4}$. Même si le Bellum ciuile ne mentionne à cette occasion que les comices romains et le rituel albain sans évoquer celui de Lavinium, c'est à mon avis cette dernière étape du parcours rituel qui explique la relative durée du séjour romain de César ${ }^{5}$. Ainsi la liaison entre les cérémonies du Capitole et du mont Albain est-elle illustrée dans le cas de Flaminius, tandis que celle qui unit les rituels albains et lavinates apparaît dans l'exemple de César.

Autre caractéristique de cet ensemble spatio-rituel : le lien entre ces trois lieux est établi, par le rite, sur base temporelle. La cérémonie du Capitole dépend de l'événement - institutionnel et de récurrence annuelle - le plus important de la vie de la res publica, l'élection consulaire, et elle consiste notamment dans la fixation de la date de la seconde festivité, célébrée sur le mont Albain; le sacrifice à faire à Lavinium - qu'on évitera d'appeler un pèlerinage, terme anachronique qu' on emploie trop souvent à son propos - est, lui aussi, déterminé par sa date, calculée en fonction de celle des Fêtes Latines du mont Albain : post diem decimum Latinarum ${ }^{6}$. Dans cet ensemble

2. Tite-Live, XXI, 63, 5-9.

3. Tite-Live, XXII, 1, 6-7: il est très probable que l'historien ne fait que reprendre ici un thème qu'il trouvait chez les annalistes.

4. César, La guerre civile, III, 2, 1 : his rebus et feriis Latinis comitiisque omnibus perficiendis XI dies tribuit. Voir Stefan Weinstock, Divus Julius, Oxford, 1971, p. 321-322.

5. Comme je l'ai soutenu dans mon livre, Alba Longa, histoire d'une légende. Recherches sur l'archéologie, la religion, les traditions de l'ancien Latium, Rome, 2008 [Bibliothèque des Écoles françaises d'Athènes et de Rome, 336], t. II, p. 580.

6. Tite-Live, VIII, 11, 15. Chez Tite-Live, qui ne fait ici que suivre la terminologie religieuse et officielle telle qu'on la trouve dans les Fastes des féries, le terme Latinae employé seul désigne le sacrifice fédéral du taureau sur le mont Albain. Il faut donc traduire : «neuf jours après le sacrifice Latin »; on reconnaît le délai religieux caractéristique qu'est la neuvaine. 
ternaire, la première étape est donc orientée vers la seconde, en fonction de laquelle est fixée la troisième; ou, pour le dire autrement, la seconde étape, celle du milieu, dépend chronologiquement de la première et commande chronologiquement la troisième, ce qui assure à la fois le bon ordre et la complétude du système.

Ainsi codifié dans l'espace et dans le temps, ce parcours cérémoniel a comme acteurs les plus hautes autorités de l'État romain: consuls, mais aussi préteurs, sénateurs et membres de différents sacerdoces. Les première et seconde étapes, à Rome et sur le mont Albain, ont probablement comme spectateurs de nombreux Romains, encore qu'il n'y ait, à notre connaissance, qu'un seul texte qui le suggère pour la seconde ${ }^{7}$ - la participation d'habitants de villes latines à la cérémonie albaine se réduisant, semble-t-il, à celle de quelques délégués par cité ${ }^{8}$. À Lavinium, par contre, les magistrats romains vont seuls, et, en tout cas, aucune source n'évoque une présence populaire. Cette implication directe des magistratures supérieures romaines dans ces trois sanctuaires n'est évidemment pas sans rapport avec le fait que non seulement le premier d'entre eux, bien sûr, mais aussi le second et le troisième sont tous placés sous le contrôle direct de l'Urbs, quand bien même ils semblent gérés par des sacerdoces de dénomination locale, dits Albanus ou Laurens Lauinas ${ }^{9}$.

\section{UN PAYSAGE NATUREL?}

Un tel parcours cérémoniel en trois temps et en trois lieux peutil constituer un paysage naturel? On pourra être tenté de répondre

7. Il s'agit de Cicéron, Ad Atticum, I, 3, 1 (datée de 67 av. J.-C.); sur l'ensemble du rituel des Feriae Latinae, on m'excusera de devoir renvoyer, pour le détail des sources antiques et leur analyse, à mon ouvrage, Alba Longa, histoire d'une légende, op. cit., t. II, p. 517-729.

8. Voir Cicéron, Pro Cn. Plancio, 9, 23, et Scholia Bobiensia, éd. Hildebrandt, Leipzig, 1907, p. 128 s., ad locum.

9. Cette conclusion a été établie dès le XIX ${ }^{e}$ siècle par l'érudition allemande : voir par ex. le célèbre article de G. Wissowa, « Die römischen Staatspriestertümer altlatinischer Gemeindekulte », Hermes 50 (1915), p. 1-33. Maria Granino Cecere et John Scheid ont fait la prosopographie de tous les titulaires connus de ces sacerdoces : voir leur étude, "Les sacerdoces publics équestres », dans Ségolène Demougin, Hubert Devijver, Marie-Thérèse Raepsaet-Charlier (dir.), L'Ordre équestre. Histoire d'une aristocratie ( ${ }^{e r}$ siècle av. J.-C. - III siècle ap. J.-C.), Rome, 1999 [Collection de l'École française de Rome, 257], p. 79-189; le développement intitulé « Funzioni sacerdotali del Lazio arcaico » est dû à Maria Grazia Granino Cecere (p. 97-113). 
par la négative, si l'on se souvient que le concept même de paysage n'est guère antique : il est venu aux Modernes par le biais de la peinture ${ }^{10}$, puis est arrivé dans les études classiques par l'archéologie, notamment suite à la pratique des prospections de surface. Mais pour répondre à cette question du paysage, il vaut la peine de s'attarder un instant sur le mons Albanus, moderne Monte Cavo. S'il n'est certes pas, contrairement à ce qu'on dit toujours, le mont le plus élevé du Latium ${ }^{11}$, il constituait, j'espère l'avoir démontré ailleurs ${ }^{12}$, le site d'Alba Longa, plus précisément, l'arx Albana mentionnée dans l'épigraphie religieuse. Il s'agit bien du relief le plus visible, dont le sommet offre un panorama étendu sur la plaine latiale, avec, au loin, les méandres du Tibre et les collines romaines, puis, barrant tout l'horizon, le rivage, où se distingue à peine l'acropole de Lavinium, et, au-delà encore, la mer immense. On est bien là sur un belvédère naturel, «lieu assez élevé où tous les objets auparavant dispersés se rassemblent sous un seul coup d'œil ». Ces mots de Fontenelle sont cités par Littré à l'article «paysage » de son Dictionnaire ${ }^{13}$ qui commence par cette définition : «Étendue du pays que l'on voit d'un seul aspect. » Pour reprendre un terme cher à Michel Foucault ${ }^{14}$, je dirais que le mons Albanus est un lieu panoptique, d'où l'on voit tout, et - à la différence de ce qu'implique ce mot - qui est vu de partout. Et pour citer cette fois une expression de Roland Barthes à propos de la Tour Eiffel, on dira que le mont est un «spectacle regardé et regardant ${ }^{15}$ ». Grâce au mont Albain, donc, étape médiane de notre parcours, Rome, Lavinium

10. Entre bien d'autres références possibles, on renverra ici à Alain Roger, Court traité du paysage, Paris, 1997.

11. Avec ses $949 \mathrm{~m}$, il le cède en effet au tout proche Maschio delle Faete, haut de $956 \mathrm{~m}$ : voir le Grande Atlante d'Italia, Istituto Geografico De Agostini, Novara, 1987, p. 357.

12. « La (re)invenzione di Alba : topografia, religione, mitografia a proposito dell'arx Albana », Scienze dell'Antichità 13, 2006 (2008), p. 749-763.

13. Dictionnaire de la langue française, Paris, 1873, t. III.

14. Cité à ce propos par Marco V. García Quintela, Le Pendu et le Noyé des Monts Albains. Recherches comparatives autour des rites et des mythes des Monts Albains, traduit de l'espagnol par Marie-Pierre Bouyssou, Bruxelles, 2007 [collection Latomus, 307], p. 131; sur ce livre, voir les réserves émises par Jacques Poucet, dans une recension à paraître dans Antiquité Classique. Je remercie son auteur de me l'avoir communiquée.

15. La Tour Eiffel, Roland Barthes, photographies André Martin, coll. «Le génie du lieu », Delpire éditeur, Berne, 1964. 
et Albe - c'est-à-dire le lieu des Féries Latines - sont, je dirais, en relation visuelle mutuelle ${ }^{16}$ : c'est pourquoi nous sommes à tous égards pleinement en droit de parler d'un paysage religieux, même si, significativement, sans doute, on n'en trouve aucune véritable description chez les auteurs anciens, compte tenu de leur discrétion habituelle dans ce domaine. Seul Virgile, en raison des nécessités de l'intrigue racontée dans l'Énéide, est amené à esquisser (XII, v. 134 à 137) ce qui est vu du mons Albanus: At Iuno e summo, qui nunc Albanus habetur/(tum neque nomen erat neque honos aut gloria monti),/prospiciens tumulo campum aspectabat et ambas/ Laurentum Troumque acies urbemque Latini. Qu'un tel point de vue panoramique soit, pour les Anciens, le privilège des dieux et non celui des hommes, on en trouve la confirmation dans les mots où Cicéron imagine Jupiter Latial contemplant un paysage albain - lacus, nemora finesque - ravagé par les constructions de Clodius. L'orateur, qui, en tant que consul avait eu pourtant à gravir le mont Albain pour célébrer les Féries, se contente, lui, d'évoquer, dans ce passage du Pro Milone $(31,85)$, les Albani tumuli atque luci, comme il le fait également dans le poème pourtant consacré à cette célébration albaine de 63 avant J.-C., en parlant des tumulos Albano in monte niualis (De divinatione, I, 11, 18). Plus tard, Juvénal décrira le même monte Cavo, vu de Lavinium, comme un sublimis apex, faisant ainsi, je crois, une subtile, mais méconnue, allusion à la coiffure rituelle des prêtres saliens dits Albani, qui officiaient sur l'arx Albana ${ }^{17}$.

Cependant, ce qui caractérise notre paysage religieux, c'est autant ce qu'il exclut que ce qu'il inclut, et c'est en cela qu'on ne saurait le réduire à un simple cadre naturel. Ainsi, bien des sanctuaires situés pourtant dans l'espace d'observation du mons Albanus ne feront pas partie du parcours cérémoniel des nouveaux consuls : par exemple, à Rome, seul le temple de Jupiter sur le Capitole est concerné, alors que, au pied du mont Albain, sub monte Albano, les bois sacrés de Ferentina (Festus 276 L) et de l'Albunea, plus loin l'Aphrodision

16. Ce que l'archéologue Camilla Briault nomme «inter-visibility »: voir «Making mountains out of molehills in the Bronze Age Aegean: visibility, ritual kits, and the idea of a peak sanctuary », World Archaeology 39 (2007), p. 122-141.

17. Juvénal, XII, v. 72, commenté dans A. Grandazzi, Alba Longa, histoire d'une légende, op. cit., p. 899. 
d'Ardée et, à Lanuvium, le temple de Junon, pourtant en situation de communio sacrorum ${ }^{18}$ avec Rome, tous ces sanctuaires, bien que présents dans la réalité physique des lieux, sont absents du paysage religieux que nous étudions. C'est dire que l'analyse de ce dernier ne relèvera ni de la géographie, fût-elle historique ${ }^{19}$, ni de l'archéologie, fût-elle spatiale ou processuelle. Or les rares études qui ont pu être consacrées à notre paysage sont de cet ordre : l'une, due au géographe Claudio Cerreti en 1987, aboutissait à un classement des sanctuaires latins en fonction de leur altimétrie ${ }^{20} ;$ l'autre, due à 1'archéologue Andrea Zifferero ${ }^{21}$, et datée de 2002, a surtout pris en compte leur plus ou moins grand éloignement de Rome, de façon à reconstituer les différents modes de contrôle du territoire par l'Urbs que révèle leur répartition géographique. Il ne manque pas non plus d'études où se trouve soulignée la relation qu'entretient tel ou tel sanctuaire particulier avec le paysage qui l'environne : les temples d'Hercule à Tibur, de la Fortune à Préneste, de Diane à Nemi bénéficient de ce point de vue d'un intérêt renouvelé22 . On considère d'ordinaire qu'un sanctuaire de hauteur traduit, de la part de la cité qui le construit ou le contrôle, une manière de s'assurer la possession du territoire environnant. Mais ici, il est indubitable que, pour le pouvoir romain de la fin de la République et de l'Empire, la conquête du Latium n'était plus depuis longtemps une question d'actualité! Il doit donc s'agir de bien autre chose : le parcours cérémoniel des nouveaux hauts magistrats romains délimite un paysage religieux d'ordre culturel beaucoup plus que naturel, que l'on se place sur le plan de la géographie ou même sur celui de l'histoire. Malgré les apparences, le paysage religieux ne résulte pas ici d'une donnée immédiate de la perception; l'espace, peut-être, si l'on en croit

18. Voir Maria Grazia Granino Cecere, art. cit., p. 109.

19. Sur les évolutions récentes de la géographie historique, voir par ex. Angelo Torre, «Un "tournant spatial” en histoire? Paysages, regards, ressources », Annales. Histoire, Sciences, Société 63 (2008), p. 1127-1144. Mais on lira d'abord les réflexions de Jean-Robert Pitte, que je remercie pour son aide sur cette question : Géographie culturelle, Paris, 2006, p. 12 sqq.

20. «Assetto territoriale e religione nel Lazio protostorico. Note preliminari per una ricerca », Rivista Geografica Italiana 94 (1987), p. 1-29.

21. "The Geography of the Ritual Landscape in Complex Societies ", dans Peter Attema et alii (dir.), New Developments in Italian Landscape Archaeology, B.A.R. International Series, 1091, Oxford, 2002, p. 246-265.

22. Selon une perspective d'étude ouverte notamment par Filippo Coarelli, I Santuari del Lazio in età repubblicana, Rome, 1987. 
$\mathrm{Kant}^{23}$ et une partie de la neurobiologie moderne ${ }^{24}$, mais le paysage, non. Une première conclusion est ainsi acquise ici : une religion produit le paysage qui lui est propre, et ce paysage est la condition de son existence en tant que religion.

\section{UNE REMONTÉE VERS L'ORIGINE ?}

Notre paysage résulte d'une composition réfléchie, abstraite a-t-on envie de dire; c'est une « còsa mentale », qui doit exprimer, vu ses protagonistes, une manière d'idéologie politique. Dans ce domaine, les noms des deuxième et troisième étapes indiquent clairement la direction: Albe La Longue et Lavinium, chacun le sait, étaient, ou plutôt passaient pour avoir été les deux métropoles originaires de Rome. Pour déchiffrer le paysage religieux où elles figurent, on peut donc, sans faute de méthode, se tourner vers le récit des origines romaines, des primordia Urbis, tel qu'il était sans cesse répété à la fin de la République et au début de l'Empire. À satiété, l'érudition - par exemple Varron -, l'histoire - par exemple TiteLive -, la poésie - par exemple Virgile -, mais aussi l'architecture - par exemple le Forum d'Auguste -, tous les secteurs de l'expression publique et idéologique du début du Principat répéteront qu'Énée, arrivé de Troie en Italie, passerait trois ans à Lavinium, après lesquels son fils Ascagne fonderait Alba Longa où régneraient, pendant trois ou quatre siècles, des rois appelés Siluii, avant que, finalement, tout puisse vraiment commencer avec la fondation de Rome. Dans cette partie du récit des origines, si connue soit-elle, quelques détails paysagers n'ont pas assez retenu l'attention : il y a une certaine logique des lieux à ce que le mythe d'Énée, héros arrivé par mer, se soit fixé en priorité à Lavinium, site côtier et même portuaire dans l'Antiquité; d'autre part, la figure de l'Ascagne grec, aux deux noms dont l'un était celui d'un lac, avait tout ${ }^{25}$ pour être réinterprétée à la romaine comme un Ascanius qui serait aussi un

23. Critique de la raison pure, traduction et présentation par Alain Renaut, Paris, (1997) 2001², p. 117 sqq. : Première partie : «L'Esthétique transcendantale ». Voir par ex. p. 129 : « tous les phénomènes extérieurs sont dans l'espace et sont déterminés a priori selon les rapports spatiaux, je veux dire à partir du principe du sens interne de manière tout à fait universelle ».

24. Voir Jean-Pierre Changeux, L'Homme neuronal, Paris, 1983, p. 175.

25. Voir A. Grandazzi, Alba Longa, histoire d'une légende, op. cit., p. 745. 
Iulus, fondateur d'une cité, Alba, située au bord d'un lac. D'autres particularités, comme la poursuite de la laie miraculeuse par Énée ${ }^{26}$ ou les allers et retours des Pénate ${ }^{27}$ entre Lavinium et Albe, peuvent être considérées comme l'étiologie de la route qui, de haute antiquité, reliait les deux sanctuaires ${ }^{28}$.

L'essentiel pour notre propos me paraît cependant être le caractère qu'a cette tradition mythique : on peut discuter sur la plus ou moins grande proximité des écrivains que j'ai nommés avec le pouvoir romain, mais il est sûr que nous avons là affaire à une véritable doctrine officielle, ce qui peut expliquer qu'elle n'ait que peu évolué sous l'Empire. Certes, je n'ignore pas l'existence de nombreuses versions ${ }^{29}$, variantes et déviantes dans le récit des origines, mais il me semble plus important de relever ici la constance, tout au long de la période impériale, de la séquence Lavinium-Albe-Rome, qui structure le mythe romain des primordia Urbis.

\section{MYTHE ET RITE}

Dans ces conditions, je crois que l'on peut considérer ce récit, sans cesse répété, comme le commentaire, l'exégèse du parcours cérémoniel qui en reprend si exactement les différentes étapes : bref, comme le mythe du rite ternaire incombant aux nouveaux hauts magistrats de Rome à leur entrée en charge. Je sais bien que cette juxtaposition du mythe au rite n'est plus à l'honneur dans nos

26. Fabius Pictor, fg. 5a Ch. (= Diod. VII, 5, 4-5); commenté par Hans Beck et Uwe Walter, Die Frühen Römischen Historiker, Darmstadt, 2001, t. I, p. 67.

27. Denys d'Halicarnasse, Antiquités romaines, I, 67, 2-4; M. Valerius Maximus, I, 8, 7; OGR, XVII, 2 et 3; Tzetzès, ad Alex., 1232.

28. Il y a, bien sûr, d'autres étiologies possibles : Yan Thomas a ainsi expliqué la répétition de l'aller et retour, sur le modèle d'une émancipation inaboutie, où manquerait la troisième répétition, de sorte que le mythe signifie le maintien - et donc le renforcement - du lien d'ancestralité de Rome avec Lavinium : Yan Thomas, " Origine » et « Commune patrie ». Étude de droit public Romain (89 av. J.-C. - 212 ap. J.-C.), Rome, 1996 [Collection de l'École française de Rome, 221]. Pour Clifford Ando, The Matter of the Gods, Berkeley/Los Angeles/London, 2008, p. $113 \mathrm{~s}$. et p. 148, l'historiette était simplement destinée à illustrer l'idée de limites de l'action humaine en matière religieuse : mais il y a deux, c'est-à-dire trois moins un, allers et retours, et c'est là ce qu'il faut expliquer.

29. Voir l'étude classique de Tim J. Cornell, «Aeneas and the Twins : the development of the Roman foundation legend », Proceedings of the Cambridge Philological Society 21 (1975), p. 1-32. 
études et a été souvent, et à juste titre, mise en procès ${ }^{30}$. Mais ici, ce qui légitime et, je crois, nécessite ce rapprochement, c'est bien une inspiration commune, faisant qu'à un rite d'État répond un mythe d'État. À la base de cette correspondance, il y a le fait, bien connu mais dont on ne tire pas toujours toutes les conséquences nécessaires, que le milieu dirigeant à Rome a une double compétence, civile et religieuse. De la sorte, pour le rite comme pour le mythe, les acteurs de l'un et les auteurs (en tout cas, les scripteurs) de l'autre sont les mêmes ou gravitent dans les mêmes sphères ${ }^{31}$ : Fabius Pictor et Caton l'Ancien, dont le rôle dans la codification écrite de la vulgate des origines romaines apparaît comme majeur, furent des hauts magistrats qui eurent à accomplir le parcours cérémoniel ici analysé; Varron fut lié à Pompée, puis ne fut pas laissé de côté par César; Virgile et Tite-Live furent proches d'Auguste.

D'un côté, cependant, nous avons, dans le récit mythique, une séquence Lavinium-Albe-Rome; de l'autre, dans le parcours rituel, une séquence Rome-Albe-Lavinium. Est-ce à dire qu'il y aurait contradiction entre les deux? Après l'avoir $\mathrm{cru}^{32}$, je ne le pense plus aujourd'hui. Car le rite réalise à rebours, en allant du plus proche, topographiquement, au plus lointain, ce que le mythe raconte, linéairement et chronologiquement. Les consuls et les hauts magistrats, en partant en dehors de Rome, vont vers l'avant de la fondation de l'Urbs, vers Albe et Lavinium, c'est-à-dire non seulement vers les lieux mais aussi vers les temps de l'origine. On est ainsi en présence d'une fiction religieuse et institutionnelle, et ce n'est sans doute pas un hasard si le seul savant à l'avoir vraiment déchiffrée comme telle était un juriste: Yan Thomas, qui avait bien analysé ce parcours cérémoniel comme « une remontée dans le temps », et comme « une quête régressive propre aux processus généalogiques $»^{33}$. Il propo-

30. Voir John Scheid, «Cultes, mythes et politique au début de l'Empire», dans Fritz Graf (dir.), Mythos in mythenloser Gesellschaft. Das Paradigma Roms, Stuttgart/Leipzig, 1993 [Colloquium Rauricum, 3], p. 109-130 (p. 115).

31. Aucune magistrature n'est attestée pour Fabius Pictor, mais en tant que patricien il dut participer au rituel; Caton fut consul en 195 av. J.-C. et censeur en 184; Varron fut tribun de la plèbe, préteur puis deux fois propréteur, et donc participant lui aussi aux cortèges sacrés. Sur le détail de leurs carrières, voir Thomas Robert Shanno Broughton, The Magistrates of the Roman Republic, t. I et II, New York, 1951 et 1952, ad locum.

32. A. Grandazzi, Alba Longa, histoire d'une légende, op. cit., p. 907.

33. Y. Thomas, "Origine" et "Commune patrie». Etude de droit public Romain (89 av. J.-C. - 212 apr. J.-C.), op. cit., chap. 6: «L'origo rituelle de 
sait en effet de relier cette triplicité topographique avec la norme des trois générations (une plus deux) régissant, à Rome, le droit successoral, qu'il s'agisse d'ascendance ou de descendance. À vrai dire, plus que cette séquence ternaire, l'intéressait le face-à-face Rome-Lavinium, qu'il interpréta comme une illustration symbolique du concept romain de l'origo, selon un processus combinant l'antériorité et l'extériorité par rapport à l'Urbs; le tout à partir de l'hypothèse d'une étape à Lavinium se faisant juste après celle du Capitole, mais qui reste fragile ${ }^{34}$. Pour ma part, je pense que, sans négliger Lavinium, il faut centrer l'analyse sur le triptyque qu'elle forme avec Albe et Rome, car l'originalité de cette séquence paysagère tient précisément à son indissolubilité, et je dirais à son insécabilité rituelle. On verra ainsi que la définition de Lavinium comme étant " en position d'origine », ou « en position d'ascendance ultime » doit être, sinon contredite radicalement, du moins assez nettement amendée et complétée.

\section{ESPACE/TEMPS}

Désormais, nous comprenons à quoi sert notre paysage religieux : à transformer, à transmuter du temps en espace et de l'espace en temps. Pour les Romains des époques républicaine et impériale, Albe et Lavinium symbolisent à elles seules les temps antérieurs à l'Urbs et surtout, à elles seules, les causes suffisantes et nécessaires de son surgissement : parce qu'elles sont des lieux géographiques résumées à deux et sans doute trois hauts reliefs, car je croirais volontiers qu'à Lavinium le temple des Pénates était sur l'acropoleelles ont pour principale qualité d'être toujours là. De ce point de vue, on a une spatialisation de la temporalité beaucoup plus que

Rome » (p. 133-193 : p. 159 et p. 133 et 168). Voir aussi son étude, «L'institution de l'origine. Sacra Principiorum Populi Romani », dans Marcel Detienne (dir.), Tracés de fondation, Paris, 1990, p. 143-170.

34. La question est en effet de savoir si ce sacrifice initial des consuls aux Pénates de Lavinium est à identifier avec le renouvellement annuel du foedus entre cette cité et Rome, fixé, précisément, neuf jours après les Féries Latines (voir supra n. 6). On répond en général par l'affirmative : voir Annie Dubourdieu, Les Origines et le développement du culte des Pénates à Rome, Rome, 1989 [Collection de l'École française de Rome, 118], p. 355-361. Réponse attentiste, mais plutôt négative, par Maria Grazia Granino Cecere, art. cit., p. 109-112. 
l'inverse. Ce primat, dans l'Antiquité, de l'espace sur le temps, on le retrouve ailleurs qu'à Rome, et ce n'est sans doute pas un hasard s'il caractérise alors toutes les mnémotechniques depuis l'archaïsme grec $^{35}$. Les causes en sont certainement multiples, au-delà d'une perception première, qui a son importance : le temps passe, l'espace reste $^{36}$. A pu jouer aussi, à mon avis, l'absence d'un comput chronologique universel, ainsi que l'importance, au moins depuis Servius Tullius ou Clisthène, du droit du sol par rapport au droit du sang. Partout, en Grèce comme à Rome, les lieux sont (ou ont vocation à être) les points d'ancrage de la durée. À cet égard, le mont Albain et Lavinium sont les hauts lieux de la Mémoire de Rome : c'est là où a commencé le commencement du monde qu'est pour les Romains la naissance - en tout cas la gestation! - de Rome. C'est là où, chaque année, par les rites complémentaires de l'indiction des Féries, du sacrifice Latin, et du sacrifice aux Pénates à Lavinium, l'origine peut, de nouveau, avoir lieu, là où le commencement doit, selon une coutume imprescriptible, être sans cesse recommencé. Le paysage religieux actualise ainsi le mythe d'origine de la cité romaine qui le met en scène, il le réifie et l'objective en l'extériorisant par la spatialisation.

\section{UNE ANALYSE EN DIACHRONIE}

Si l'analyse synchronique peut, de la sorte, éclairer le fonctionnement de ce système spatio-rituel - on le voit, extrêmement cohérent -, le détail de son ordonnancement reste cependant inexpliqué. Pourquoi pas, après tout, une autre successivité, qui aurait été : Rome-Lavinium-Albe? C'est l'analyse diachronique qui, je crois, permettra de répondre à cette dernière interrogation, tant il est vrai qu'un paysage religieux forme un discours qui, en tant que tel, doit être interrogé à la croisée du synchronique et du diachronique. La succession Lavinium-Albe-Rome trouve-t-elle son origine dans un différentiel chronologique réel entre les trois sites? Le relativisme post-moderne, qui fait florès aujourd'hui, notamment dans les uni-

35. Voir Frances Amelia Yates, L'Art de la mémoire, trad. Daniel Arasse, Paris, 1975, p. 13 s. p. 391.

36. J'emprunte cet aphorisme à Gérard Genette, Bardadrac, Paris, 2006, 
versités anglo-saxonnes, répondra fermement par la négative. Il est troublant, cependant, de constater que, parmi les spécialistes actuels de la protohistoire latiale, plusieurs archéologues éminents, si différentes soient leurs orientations respectives, apportent, eux, une réponse positive à cette question, en validant ainsi factuellement le schéma proposé par le système spatio-rituel étudié ici : par exemple, Giovanni Colonna ${ }^{37}$, Anna-Maria Bietti Sestieri et Anna De Santis ${ }^{38}$ se rejoignent au moins sur ce point important. On peut évidemment admettre qu'un système rituel puisse à la fois garder trace de réalités très anciennes et impliquer une symbolique beaucoup plus récente. Toutefois, à la réflexion, je me demande si ces archéologues n'ont pas, malgré qu'ils en aient, subi l'influence de la tradition littéraire antique et des travaux de leurs prédécesseurs qui reposaient implicitement un peu sur elle. Il me semble, en particulier, que la mise en lumière, actuellement en cours, d'importantes présences archéologiques remontant au Néolithique et à l'âge du Bronze, au cœur même du massif albain ${ }^{39}$, pourrait conduire à mettre en question ce schéma traditionnel. D'autant plus que d'autres considérations, tirées de l'étude des traditions légendaires, montreraient plutôt que le triptyque classique résulte d'une manipulation mythique opérée par Rome ${ }^{40}$, comme le révèlent également deux indices qui n'ont,

37. «Preistoria e protostoria di Roma e del Lazio », dans Massimo Pallottino et alii (dir.), Popoli e Civiltà dell'Italia Antica, II, Rome, 1974, p. 273-346 (p. 296, 302 s., 328, 330-331); id., " I Latini e gli altri popoli del Lazio », dans Giovanni Pugliese Caratelli (dir.), Italia, omnium terrarum alumna, Milan, 1988, p. $441-528=i d$., Italia Ante Romanum Imperium. Scritti di antichità etrusche, italiche e romane (1958-1998), Rome, 2005, t. I, 2, p. 547-654 (p. 555 s. et 561).

38. " Relative and absolute Chronology of Latium Vetus from the Late Bronze Age to the Transition to the Orientalising Period », dans Dirk Brandherm et Martin Trachsel (dir.), A New Dawn for the Dark Age? L'âge obscur se fait-il jour de nouveau? Les paradigmes changeants de la chronologie de l'âge du Fer en Méditerranée, Union int. des sciences préhist. et protohistoriques, Actes du $\mathrm{XV}^{\mathrm{e}}$ Congrès mondial, B.A.R. International Series, 1871, Oxford, 2008, p. 119-133.

39. Voir notamment la publication récente, par Luciana Drago Troccoli, d'une hache et des vestiges d'une puissante enceinte, le tout identifié sur le relief dit Monte dei Ferrari, au s.-e. du massif : « Considerazioni sul popolamento del settore orientale dei Colli Albani alla luce delle recenti ricerche nell'area dell'Artemisio ", RPAA 75 (2002-2003), p. 33-104 (p. 44 s.).

40. Et datable, à mon avis, des lendemains de la victoire remportée en 338 av. J.-C. sur la Ligue latine : voir Alexandre Grandazzi, « Le roi Latinus : analyse d'une figure légendaire ", CRAI, 1988, p. 481-497; Andrea Carandini parlera ensuite d'une «ricontestualizzazione mitica» qu'il datera de l'ère archaïque : La Nascita di Roma. Dèi, lari, eroi e uomini all'alba di una civiltà, Turin, 1997, p. 103-112. 
de ce point de vue, pas retenu l'attention ${ }^{41}$. Dans ces conditions, la précellence accordée à Lavinium par le rite romain ne signifierait pas nécessairement la reconnaissance de la supériorité réelle qu'aurait eue jadis cette cité, ou du moins ce territoire, sur le reste de l'ancien Latium. La religion, qui, souvent, constitue un excellent conservatoire des réalités de civilisation les plus anciennes, montrerait au contraire que les festivités du mont Albain avaient été, et de loin, la panégyrie majeure des peuples latins ${ }^{42}$, qui venaient y renouveler leur alliance mutuelle par le partage sacrificiel, entre leurs dirigeants, des parts du taureau blanc offert à Jupiter Latial. C'est bien la raison pour laquelle Rome qui, au moins par sa langue et sa religion, appartenait à cet ensemble, n'eut de cesse de prendre le contrôle de ces Féries Latines. Sans doute peut-on présumer qu'il y eut même une époque où elle se réclamait directement du centre albain, afin de justifier ses prétentions à l'hégémonie en Latium. Il se pourrait même qu'un document exceptionnel garde la trace d'une telle situation : l'orientation albaine de la Forma Urbis, remise récemment en lumière par Emilio Rodríguez Almeida ${ }^{43}$, illustre un face-à-face Rome-Albe, d'où Lavinium est absente. Par son caractère augural, par la centralité qu'il donne à l'arx et au Capitole, où l'on sait maintenant que le temple de Jupiter remonte bien à l'époque royale ${ }^{44}$, le rapport direct Rome-Albe, qui est à la base d'un tel document et qui l'a évidemment précédé de beaucoup, nous paraît remonter à l'époque archaïque, disons aux Tarquins. On voit que dans le parcours rituel des consuls, cette relation directe n'a pas disparu; mais elle a été, si je puis dire, minorée, grâce à la promotion de Lavinium au premier plan. C'est de Lavinium que seraient censés provenir les Pénates de Rome, tandis que l'ancienne panégyrie latine et albaine, où désormais chaque peuple latin aurait à prier pour le peuple romain,

41. Voir infra ce qui est dit de la F.U.R. et d'Alba Fucens.

42. Dont la liste la plus ancienne est donnée par Pline l'Ancien : voir notre étude, «La liste plinienne des populi dits Albenses (Histoire Naturelle, III, 69) : anciennes et nouvelles hypothèses ", REL 77 (1999), p. 30-49.

43. Formae Urbis Antiquae. Le mappe marmoree di Roma tra la Repubblica e Settimio Severo, [Collection de l'École française de Rome, 305], Rome, 2002, fig. 4, p. 12.

44. Voir Anna Mura Sommella Mura, " "La grande Roma dei Tarquini”". Alterne vicende di una felice intuizione ", dans F. Roscetti (sous la dir. de), Il Classico nella Roma contemporanea : mito, modelli, memoria, Rome, 2002, t. II, p. 303-323. Toutefois, selon Pier Luigi Tucci (dans JRA 19 (2006), p. 389-390), les proportions attribuées maintenant au sanctuaire sont sans doute exagérées. 
deviendrait le modèle du type d'alliance et d'intégration que l'Urbs souhaitait proposer au monde. À quel moment a pu être opéré cet ajustement, ce renversement, à la fois rituel et mythique? Ou, pour le dire autrement, à quel moment le mythe d'Énée à Lavinium estil devenu un mythe d'État pour Rome? Avec beaucoup d'autres ${ }^{45}$, je propose 338 avant J.-C., c'est-à-dire le moment où Rome vainc et dissout la vieille Ligue Latine, dont le mont Albain avait été le centre religieux, et sans doute politique aussi dans les phases les plus anciennes. Mais, de cette chronologie classique, une preuve nouvelle peut être présentée, qui permet de réfuter les chronologies plus hautes souvent proposées aujourd'hui, et qui est précisément de l'ordre du paysage.

Quelques années après la dissolution du nomen Latinum, en effet, les Romains fondèrent, en 303 avant J.-C., aux marches du Latium et pour en défendre l'accès, la colonie d'Alba Fucens, dont le site, comme celui de sa devancière mythique, se caractérisait par le voisinage de reliefs et d'un lac, et dont le nom double montrait qu'ils n'oubliaient pas Alba Longa, conclusion qui vaut aussi bien dans l'hypothèse où le toponyme Alba aurait déjà été présent sur place, que dans celle où il aurait été apporté par les fondateurs de la nouvelle cité. Or, puisque, dans le mythe, Alba Longa était fondée par Ascagne, fils d'Énée qui avait régné à Lavinium, fonder une Alba Fucens, c'était, de la part des Romains, s'affirmer comme des Aeneades, c'est-à-dire des héritiers d'une Lavinium désormais définie comme métropole d'Albe. Inspiration mythique qui n'empêchait pas, bien sûr, la colonie de se présenter, dans sa réalité topographique et monumentale, comme une copie de Rome ${ }^{46}$. C'est donc à ce moment, à mon avis, qu'est mis en place le triple parcours cérémoniel dont Lavinium est à la fois l'aboutissement dans la topographie et le point de départ dans la mythographie. Il n'est ainsi pas indifférent que la précision sur l'intervalle de temps séparant, dans notre parcours rituel, les étapes albaine et lavinate, apparaisse, dans

45. Notamment Ferdinando Castagnoli, «La leggenda di Enea nel Lazio », Studi Romani 30 (1982), p. 1-15.

46. Selon ce qu'a montré Mario Torelli, « Il "diribitorium" di Alba Fucens e il "campus" eroico di Herdonia », dans Jozef Mertens, Roger Lambrechts (dir.), Comunità indigene e problemi della romanizzazione nell'Italia centro-meridionale (IV-III sec. av. C.), Bruxelles/Rome, 1991, p. 39-63. 
le récit livien ${ }^{47}$, précisément à propos de la paix de 338 avant J.-C. Quant à la fameuse mention de sacra quaedam in monte Albano Lauiniique que le même Tite-Live $(\mathrm{V}, 52,8)$ place dans la bouche de Camille s'adressant aux Romains tentés de s'installer à Véies, il s'agit très probablement d'une simple anticipation due à l'historien, l'ensemble du discours étant du reste une fiction.

\section{LE PAYSAGE RELIGIEUX DU SUBURBIUM COMME DISCOURS SUR L'IDENTITÉ ROMAINE}

Chacune de ces trois étapes fait donc sens : celle du Capitole reconnait la supériorité, la maiestas du Sénat et du peuple de Rome; celle des Féries dites Latines illustre, par une métonymie à la fois spatiale et temporelle, le modèle de toute alliance future; il faut ici se souvenir que Latium n'est pas seulement un toponyme mais aussi, à partir de 89 avant J.-C. au moins, un concept juridique ${ }^{48}$ : parce qu'ils avaient été les premiers à être conquis et absorbés, les Latini et le Latium allaient rester le paradigme ${ }^{49}$ de toute intégration à venir; l'étape de Lavinium, enfin, retrouve les dieux qui font l'identité de Rome, les Pénates d'Énée. C'est bien pourquoi, Lavinium n'est pas « en position d'ascendance ultime ». On l'a dit ${ }^{50}$, mais c'est oublier que ses Pénates viennent d'ailleurs, viennent de Troie. À mon sens, l'exterritorialité caractérisant le parcours cérémoniel qui, de Rome, conduit d'abord à Albe puis à Lavinium, port et lieu de contact avec l'outre-mer, ne peut signifier qu'une chose : le refus romain de l'autochtonie ${ }^{51}$. Bien entendu, on peut penser que les lendemains de la Guerre Sociale et l'intégration accélérée

47. VIII, 11, 15 : cum Laurentibus renouari foedus iussum renouaturque ex eo quotannis post diem decimum Latinarum.

48. Voir Pline, Histoire Naturelle, IV, 30 ; Tacite, Histoires, III, 55, commentés par David Kremer, Ius Latinum. Le concept de droit latin sous la République et l'Empire, Paris, 2006, p. 111.

49. Comme le souligne, à propos de leur mention dans les Actes des Jeux Séculaires, Alison E. Cooley, « Beyond Rome and Latium : Roman religion in the age of Augustus ", dans Celia E. Schultz et Paul B. Harvey Jr. (dir.), Religion in Republican Italy, Cambridge, 2006, p. 228-252 (p. 229 et p. 235-236).

50. Y. Thomas, "Origine" et "Commune patrie ». Étude de droit public Romain (89 av. J.-C. - 212 apr. J.-C.), op. cit., p. 134.

51. Revendiquée par contre par Athènes : voir Nicole Loraux, Né de la Terre. Mythe et politique à Athènes, Paris, 1996. 
des populations d'origine italique qui en fut la conséquence paradoxale, allaient donner au modèle ainsi créé une portée beaucoup plus vaste, que l'Empire élargirait encore. De ce point de vue, ce n'est peut-être pas seulement le hasard de la documentation qui fait que la titulature sacrale Laurens Lavinas apparaît épigraphiquement durant le règne de Claude $^{52}$, autrement dit d'un empereur qui œuvra décisivement pour rendre la citoyenneté romaine accessible à de nouvelles élites provinciales ${ }^{53}$. Quoi qu'il en soit, la spatialisation réalisée par ce paysage religieux avait pour intérêt de rendre immédiatement visible aux masses le modèle d'intégration ainsi proposé. Toutefois, elle n'était pas exportable, dépendant d'un paysage par définition immobile. C'est dire toute l'importance que pouvaient prendre dans cette perspective des œuvres littéraires comme l'Énéide ou l'Histoire de Tite-Live : elles n'ont pas créé le système spatio-rituel ici analysé, mais elles l'auront popularisé dans tout 1'Empire. À cet égard, l'Énéide - dont les sept premiers vers de prologue ${ }^{54}$ nomment, dans l'ordre, Troie, Lavinium, Albe et Rome - est bien le poème de l'identité romaine ${ }^{55}$; et on comprend mieux pourquoi - ce qui a toujours un peu gêné les commentateurs les $A b$ Urbe condita libri, et, avant eux, les récits annalistiques, commencent, non avec Romulus, mais bien avec Éné ${ }^{56}$.

Au total, à quoi peut donc servir un paysage religieux? À fabriquer du temps, et du temps d'origine, indéfiniment renouvelable; à rendre, par une espèce d'ars memoriae en acte, l'histoire visible,

52. Peut-être à l'occasion des Ludi Saeculares de 47, les Libri Sibullini étant mentionnés en CIL X, 797 : voir Alison E. Cooley, « Politics a. religion in the Ager Laurens », dans ead. (dir.), The Epigraphic Landscape of Roman Italy, Londres, 2000 [Bulletin of the Institute of Classical Studies, Supplement 73], p. 173-191, qui définit la succession des cérémonies sur le mont Albain et à Lavinium comme « a delicate compromise » (p. 176) entre les prétentions de deux centres jadis rivaux : c'est méconnaître la sémiotique chrono-spatiale mise en œuvre par ce parcours rituel.

53. Pour une interprétation d'ensemble de cette politique, on lira les denses réflexions d'Andrea Giardina, L'Italia romana. Storie di un'identità incompiuta, Rome/Bari [1997] 2004, p. 3-116.

54. V. 1 : Troiae; v. 2-3 : Lauiniaque ... litora; v. 7 : Albanique patres atque altae moenia Romae. À Lavinium, lieu de débarquement, et à Albe qui n'est qu'une ascendance, ces vers opposent la protection offerte par Rome.

55. Voir Katharine Toll, « Making Roman-ness and the Aeneid », Classical Antiquity 16 (1997), p. 34-56.

56. À vrai dire, en hommage à ses propres origines, l'historien padouan consacre, comme on le sait, les premières lignes de son premier livre $(I, 1,1)$ à Anténor, dont le nom vient toutefois en second après celui d'Énée. 
tangible presque, à la transformer, à la récrire pour la faire devenir Mémoire collective ${ }^{57}$; à penser le rapport à l'autre; à construire une identité ouverte, car sans cesse en devenir. Pouvoir recommencer sans cesse, c'est pouvoir continuer sans fin: imperium sine fine. Telle est la logique profonde mise en œuvre, dans un système spatio-temporel où mythe et rite se répondent exactement, par un impérialisme romain sûr de lui, et dominateur dans la mesure même où il se veut intégrationniste.

C'est pourquoi on peut, je crois, lire ce paysage religieux RomeAlbe-Lavinium comme un discours muet, comme une espèce de gigantesque métaphore chosifiée. Significativement, le droit antique décrit en termes topographiques ce concept juridique qu'estl' acquisition de la citoyenneté romaine : in/ad ciuitatem Romanam uenire ${ }^{58}$. Ce que proclame alors ce paysage-métaphore, c'est ceci : le monde entier a vocation à aborder, un jour, sur les côtes de l'Italie, puis à gravir ensemble le mont de l'alliance fédérative qu'est le Latium, et, enfin, à se retrouver, pour finir et pour commencer, à Rome.

Université Paris IV-Sorbonne/E.A.1491

1, rue Victor Cousin

75230 PARIS cedex 05

alexandre.grandazzi@orange.fr

57. On pense bien sûr à Maurice Halbwachs, La Mémoire collective [1950], édition critique établie par Gérard Namer, Paris, 1997 (voir p. 227-236) : mais ici, il s'agit d'une idéologie d'État, élaborée par une élite dirigeante tout à fait consciente des objectifs qu'elle poursuit.

58. Festus 155 L, Municipium. 